Editorial

Z Gerontol Geriat 2020 · 53:203-204

https://doi.org/10.1007/s00391-020-01706-5

(c) Springer Medizin Verlag $\mathrm{GmbH}$, ein Teil von Springer Nature 2020

Das Alter(n) ist vielfältig. Über diese Aussage herrscht in der Gerontologie weitgehende Einigkeit. Auch in der Politik ist das Bewusstsein dafür, dass es sich bei den Älteren nicht um eine homogene Gruppe mit einheitlichen Interessen und Bedürfnissen handelt, in den vergangenen Jahrzehnten gewachsen. Und in der Gesellschaft finden sich in Filmen, Büchern und Zeitungsartikeln zunehmend vielfältige Bilder älterer Menschen. Zugleich sind negative Altersbilder und Stereotype weiterhin wirksam und Altersdiskriminierung wird häufig noch nicht einmal als solche erkannt und benannt.

Allerdings handelt es sich bei der „Vielfalt des Alters" nicht um ein theoretisches Konzept, sondern um eine rein beschreibende Begrifflichkeit. Ihr kommt damit auch keine analytische Funktion zu und sie sagt nichts über die Art der Vielfalt, ihr Zustandekommen oder ihre Wirkung. Vor allem blendet die Rede von der Vielfalt des Alters aus, dass es sich überwiegend nicht um horizontale Unterschiede von selbstgewählten Lebensstilen handelt, sondern um strukturelle Ungleichheiten, die mit dauerhaften, oft biografisch verankerten Ausschlüssen, Abwertungen und Benachteiligungen verbunden sind. Strukturelle Machtund Ungleichheitsverhältnisse drohen damit aus dem Blick zu geraten. Auch das Zusammenwirken mehrerer gesellschaftlicher Ungleichheitsachsen entlang von Alter, Geschlecht, Klassen oder Herkunft kann mit dem Begriff der Vielfalt nicht angemessen erfasst, untersucht und beschrieben werden.

Für die wissenschaftliche Untersuchung der Vielfalt des Alter(n)s bietet sich das Konzept der Intersektionalität an, in dem die Überschneidung unterschiedlicher Ungleichheitsachsen

\author{
Anna Sarah Richter ${ }^{1}$ Cornelia Kricheldorff ${ }^{2}$ \\ ' Deutscher Verein für öffentliche und private Fürsorge, Berlin, Deutschland \\ ${ }^{2}$ Soziale Gerontologie und Soziale Arbeit im Gesundheitswesen, Katholische Hochschule Freiburg, \\ Freiburg, Deutschland
}

\title{
Alter(n) und Intersektionalität
}

und die simultane Hervorbringung von Differenzverhältnissen in alltäglichen Praxen den zentralen Untersuchungsgegenstand bilden. Das Konzept hat seinen Ursprung in der Frauen- und Geschlechterforschung [1-4]. Im Kern geht es um die Anerkennung vielfältiger Differenzen zwischen Frauen und die damit verbundenen unterschiedlichen Erfahrungen von Ungleichheitsverhältnissen, die sich aus den Wechselwirkungen unterschiedlicher Zugehörigkeiten ergeben. Es wurde kritisiert, dass in der feministischen Bewegung und Forschung der 1970er-Jahre die spezifischen Erfahrungen etwa von migrantischen Frauen, „women of color" und Arbeiterinnen nicht berücksichtigt wurden. Dafür ist die universalistische Konzeption der Kategorie „Frau“ unzureichend. Entsprechend liegt der Fokus intersektionaler Forschung und Theoriebildung auf dem Zusammenwirken von geschlechtlicher Ungleichheit mit Rassismus und Klassenzugehörigkeit.

Die Kategorie des Alter(n)s fristet in der Intersektionalitätsforschung dagegen bislang ein eher randständiges Dasein. Wenn überhaupt, tritt sie als „Platzhalterkategorie“ in Erscheinung, mit der angedeutet wird, dass es neben Geschlecht, Klasse und Ethnizität weitere Kategorien gibt, die beachtet werden müssen. Andererseits kann auch für die gerontologische Forschung festgestellt werden, dass hier die intersektionale Perspektive oftmals fehlt. Im vorliegenden Themenschwerpunkt sollen die Eigenständigkeit von Alter als Differenzund Ungleichheitskategorie sichtbar gemacht, Konzepte zur Untersuchung des Zusammenwirkens von Alter und anderen Ungleichheitskategorien vorgestellt und Ergebnisse intersektionaler
Analysen diskutiert werden. Anhand unterschiedlicher Dimensionen wird aufgezeigt, wie die soziale Konstruktion von Alter(n) mit den Konstruktionen anderer Kategorien sozialer Differenzierung wie z.B. geschlechtliche und sexuelle Identität, ethnische Herkunft oder Behinderung zusammenwirkt. Dabei interessiert, welche Wechselwirkungen zwischen diesen Differenzierungskategorien bestehen oder inwiefern Marginalisierung und Abwertung aufgrund des höheren Lebensalters mit anderen Ungleichheitsdimensionen verwoben sind. Zentral ist dabei der Gedanke, dass es sich bei allen untersuchten Differenzund Ungleichheitsverhältnissen nicht um biologisch festgelegte Unterschiede handelt, sondern dass diese immer (auch) sozial erzeugt sind.

Der Beitrag von Anna Sarah Richter führt in das Konzept der Intersektionalität ein und macht auf die Mehrdimensionalität von Ungleichheitskategorien aufmerksam. Darauf aufbauend wird ein Vorschlag für eine gerontologisch nutzbar zu machende Heuristik von Intersektionalität entwickelt, die auf die Verbindung zwischen strukturellen Dimensionen gesellschaftlicher Ungleichheit und subjektivem Erleben und Handeln fokussiert. Tina Denninger fragt in ihrem Beitrag danach, wie das Zusammenwirken der Kategorien Alter(n) und Behinderung in einer machtsensiblen Analyse erfasst werden kann. Dazu verbindet sie körpertheoretische Ansätze mit dem Konzept der Normalität, um so die Theoretisierung beider Kategorien voranzutreiben. Ralf Lottmann zeigt anhand einer qualitativen Studie die Bedeutung von geschlechtlicher und sexueller Vielfalt in der Altenhilfe sowie ihre Wechselwirkungen mit weiteren Differenzierungsmerk- 
malen. Das Ergebnis zeigt, dass eine in-
tersektionale Perspektive dazu beitragen kann, eine bedarfsgerechte Versorgung im hohen Alter für LSBTI*-Senior*innen zu schaffen. Silke Migala und Uwe Flick erörtern, wie ein intersektionaler Zugang für die Analyse ethischer Normen und das Verständnis von Diversität (hinsichtlich Alter[n], Ethnizität, Religion und Geschlecht) in gesundheitspolitischen Diskursen zur pflegerischen Versorgung am Lebensende konstruktiv genutzt werden kann. So können Auslassungen im politischen Diskurs zu gerechtigkeitserheblichen Fragen sichtbar gemacht werden, die sich im Kontext einer konkreten sozialen Praxis zeigen.

In ihrer unterschiedlichen Herangehensweise zeigen die hier versammelten Beiträge die Vielfalt intersektionaler Ansätze selbst. Sie machen aber auch deutlich, wie intersektionale Perspektiven in der gerontologischen Forschung genutzt und die Theoriebildung zum Altern anregen können.

\section{Korrespondenzadresse

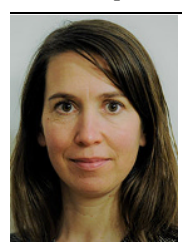 \\ Dr. Anna Sarah Richter \\ Deutscher Verein für \\ öffentliche und private \\ Fürsorge \\ Michaelkirchstr. 17/18, \\ 10179 Berlin, Deutschland \\ richter@deutscher-verein.de

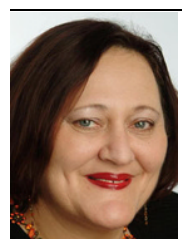 \\ Prof. Dr. \\ Cornelia Kricheldorff \\ Soziale Gerontologie \\ und Soziale Arbeit im \\ Gesundheitswesen, \\ Katholische Hochschule \\ Freiburg \\ Karlstr. 63, 79104 Freiburg, \\ Deutschland \\ cornelia.kricheldorff@ \\ kh-freiburg.de}

Interessenkonflikt. A. S. Richter und C. Kricheldorff geben an, dass kein Interessenkonflikt besteht.

\section{Literatur}

1. Combahee River Collective (1997) A black feminist statement. In: Nicholson L (Hrsg) The second wave: a reader in feminist theory. Routledge, New York, s63-70

2. Crenshaw K (1989) Demarginalizing the intersection of race and sex. A black feminist critique of antidiscrimination doctrine, feminist theory and antiracist politics. Univ Chic Leg Forum 1989:139-167

3. Lutz H, Herrera Vivar MT, Supik L (2010) Fokus Intersektionalität - Eine Einleitung. In: Lutz H, Herrera Vivar MT, Supik L (Hrsg) Fokus Intersektionalität. Bewegungen und Verortungen eines vielschichtigen Konzepts. VS, Wiesbaden, S9-30

4. Walgenbach K (2007) Gender als interdependente Kategorie. In: Walgenbach K, Dietze G, Hornscheidt A et al (Hrsg) Gender als interdependente Kategorie. Neue Perspektiven auf Intersektionalität, Diversität und Heterogenität. Budrich, Opladen, Farmington Hills, S 23-64
Aktuelle Informationen

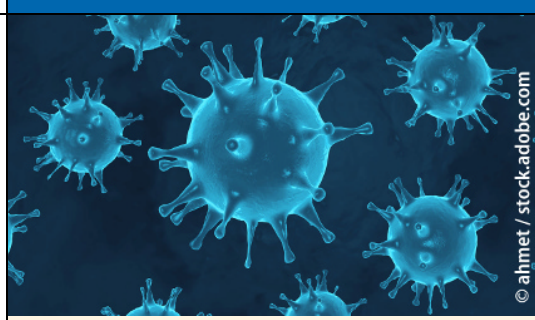

\section{Aktuelle Entwicklung zu COVID-19 bei Springer Nature und Springer Medizin}

Springer Nature und Springer Medizin unterstützen die globale Reaktion auf die COVID-19-Pandemie, indem ein schneller und direkter $\mathrm{Zu}$ gang zu den neuesten verfügbaren Forschungsergebnissen und Daten ermöglicht wird.

Auf der Homepage SpringerMedizin.de finden Sie ein immer aktuelles Dossier mit Beiträgen, Forschungsarbeiten und Ergebnissen zu SARS-CoV-2 sowie relevanten Links.

Darin z.B. auch die kürzlich publizierte

Empfehlung von DIVI, DGIIN, DGAI und DGP zur Intensivtherapie von Patienten mit COVID-19.

Springer Nature arbeitet mit globalen Organisationen zusammen, und verlinkt über SpringerNature.com/de auf eine eigene Landingpage mit einer Vielzahl an Information sowie freiem Zugriff auf die COVID-19-Contentplattformen von Nature Research, BioMed Central (BMC) und Springer.

Das Dossier zu Coronavirus / Covid-19 von Springer Medizin finden Sie hier: www.springermedizin.de/covid-19

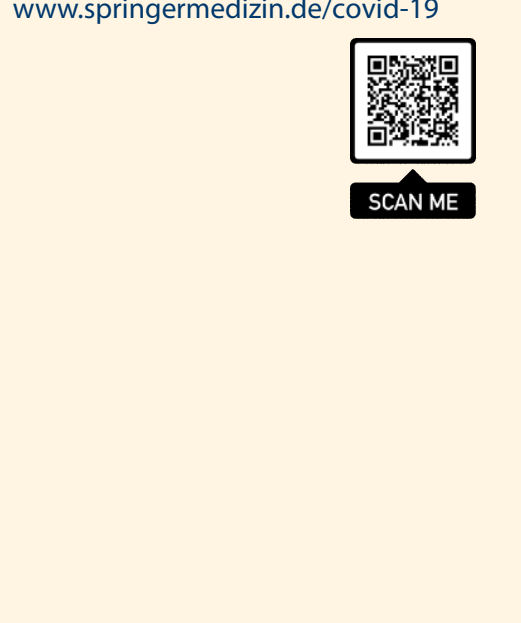

